\title{
Fixed point theorems for complex non-self mappings satisfying an implicit relation in Kaleva-Seikkala's type fuzzy metric spaces
}

\author{
Ming-Liang Song ${ }^{*}$ and Zhong-qian Wang
}

"Correspondence:

mlsong2004@163.com

Mathematics and Information

Technology School, Jiangsu Second Normal University, Nanjing, 210013, P.R. China

\begin{abstract}
In this paper, by using $\boldsymbol{A}_{\varphi}$-type real functions, some fixed point theorems for complex non-self mappings satisfying an implicit relation in two fuzzy metric spaces in the sense of Kaleva and Seikkala are established. As applications, we obtain the corresponding fixed point theorems for complex non-self mappings satisfying an implicit relation in two Menger probabilistic metric spaces. Also, an example, which shows the validity of the hypotheses of our main results, is given.
\end{abstract}

MSC: 47H10;46S40; 54E70

Keywords: fuzzy metric space; $\boldsymbol{A}_{\varphi}$-type real functions; implicit relation; fixed point; Menger probabilistic metric space

\section{Introduction}

In 1984, Kaleva and Seikkala [1] introduced the concept of a fuzzy metric space by setting the distance between two points to be a nonnegative fuzzy real number. From then on, some important results for mappings in fuzzy metric spaces, such as variational principles, coincidence theorems and various fixed point theorems, etc., were stated in subsequent work (see [2-7], etc.). It is well known that the Kaleva-Seikkala's type fuzzy metric space is an important generalization of a Menger probabilistic metric space and a metric space as well (see $[1,4,8]$ ) and possesses rich structure with suitable choices of binary operations. Recently, Huang and Wu [9] investigated the completion of the Kaleva-Seikkala' type fuzzy metric space. Previous work on this question is based on the $t$-norm min and the $t$-conorm max. Their meaningful work does away with this restriction. Shortly afterwards, Xiao et al. [10] proved some fixed point theorems of self-mapping for nonlinear contraction in a complete fuzzy metric space. Their main results do away with the restriction of the $t$ conorm max and are based on a generic class of binary operations.

On the other hand, fixed point theorems and their applications for complex non-self mappings in two metric spaces were considered by Fisher [11] in 1981. Telci [12] generalized the fixed point theorems by introducing the notion of the real functions $\mathcal{F}$. Recently, Aliouche and Fisher [13] proved two new fixed point theorems for complex non-self mappings by introducing the notion of the real functions $\mathcal{F}$ satisfying an implicit relation in two metric spaces, which is a generalization of the Telci fixed point theorem in [12].

Inspired by the work of $[9,10,13]$, in this paper we discuss the unique existence of fixed points for complex non-self mappings in two fuzzy metric spaces in the sense of Kaleva

(2013 Song and Wang: licensee Springer. This is an Open Access article distributed under the terms of the Creative Commons Attribution License (http://creativecommons.org/licenses/by/2.0), which permits unrestricted use, distribution, and reproduction in any medium, provided the original work is properly cited. 
and Seikkala. In Sections 2-3, we first introduce the new real function class $\mathcal{A}_{\varphi}$ satisfying an implicit relation. Then, by using $\mathcal{A}_{\varphi}$-type real functions, some fixed point theorems for complex non-self mappings satisfying an implicit relation in fuzzy metric spaces are established. Our main results do away with the restriction of the $t$-conorm max and are based on a generic class of binary operations. As some immediate consequences of this theorems, we obtain Theorem 3.4 and Theorem 3.5. It is a generalization of the main results in [13]. In Section 4, as applications, we obtain the corresponding fixed point theorems in Menger probabilistic metric spaces. Also, an example, which shows the validity of the hypotheses of our main results, is given.

\section{Basic concepts and lemmas}

Throughout this paper, let $\mathbb{N}$ be the set of all positive integers, $\mathbb{R}=(-\infty,+\infty)$ and $\mathbb{R}^{+}=[0,+\infty)$. For the details of a fuzzy real number, we refer the reader to Kaleva and Seikkala [1], Dubois and Prade [14], Bag and Samanta [15].

Definition 2.1 (Dubois and Prade [14]) A mapping $u: \mathbb{R} \rightarrow[0,1]$ is called a fuzzy real number or a fuzzy interval, whose $\alpha$-level set is denoted by $[u]_{\alpha}=\{t \in \mathbb{R}: u(t) \geq \alpha\}$, if it satisfies two axioms:

(1) There exists $t_{0} \in \mathbb{R}$ such that $u\left(t_{0}\right)=1$;

(2) $[u]_{\alpha}=\left[\lambda_{\alpha}, \rho_{\alpha}\right]$ is a closed interval of $\mathbb{R}$ for each $\alpha \in(0,1]$, where $-\infty<\lambda_{\alpha} \leq \rho_{\alpha}<+\infty$.

Let us denote the set of all such fuzzy real numbers by $G$. If $u \in G$ and $u(t)=0$ whenever $t<0$, then $u$ is called a nonnegative fuzzy real number, and by $G^{+}$we mean the set of all nonnegative fuzzy real numbers. If $\lambda_{\alpha}=-\infty$ and $\rho_{\alpha}=+\infty$ are admissible, then, for the sake of clarity, $u$ is called a generalized fuzzy real number. The sets of all generalized fuzzy real numbers or all generalized nonnegative fuzzy real numbers are denoted by $G_{\infty}$ and $G_{\infty}^{+}$, respectively. In that case, if $\lambda_{\alpha}=-\infty$, for instance, then $\left[\lambda_{\alpha}, \rho_{\alpha}\right]$ means the interval $\left(-\infty, \rho_{\alpha}\right]$. Since each $r \in \mathbb{R}$ can be considered as a fuzzy real number $\bar{r}$ defined by

$$
\bar{r}(t)= \begin{cases}1, & \text { if } t=r, \\ 0, & \text { if } t \neq r,\end{cases}
$$

$\mathbb{R}$ can be embedded in $G$, and $\overline{0} \in G^{+}$.

Lemma 2.1 (Xiao and Zhu [16]) Let $u \in G, \alpha \in(0,1]$, and $[u]_{\alpha}=\left[\lambda_{\alpha}, \rho_{\alpha}\right]$. Then

(1) $\lim _{t \rightarrow-\infty} u(t)=0=\lim _{t \rightarrow+\infty} u(t)$;

(2) $u(t)$ is a left-continuous and non-increasing function for $t \in\left(\lambda_{1},+\infty\right)$;

(3) $\rho_{\alpha}$ is a left-continuous and non-increasing function for $\alpha \in(0,1]$.

Definition 2.2 (Kaleva and Seikkala [1]) Let $X$ be a non-empty set, $d$ be a mapping from $X \times X$ into $G^{+}$, and let the mappings $L, R:[0,1] \times[0,1] \rightarrow[0,1]$ be symmetric, nondecreasing in both arguments and satisfy $L(0,0)=0$ and $R(1,1)=1$. For $\alpha \in(0,1]$ and $x, y \in X$, define the mapping

$$
[d(x, y)]_{\alpha}=\left[\lambda_{\alpha}(x, y), \rho_{\alpha}(x, y)\right] .
$$


The quadruple $(X, d, L, R)$ is called a fuzzy metric space (briefly, FMS), and $d$ is called a fuzzy metric on $X$ if

$(\mathrm{FM}-1) d(x, y)=\overline{0}$ if and only if $x=y$;

$(\mathrm{FM}-2) d(x, y)=d(y, x)$ for all $x, y \in X$;

(FM-3) for all $x, y, z \in X$ :

(FM-3L) $d(x, y)(s+t) \geq L(d(x, z)(s), d(z, y)(t))$, whenever $s \leq \lambda_{1}(x, z), t \leq \lambda_{1}(z, y)$ and $s+t \leq \lambda_{1}(x, y)$

(FM-3R) $d(x, y)(s+t) \leq R(d(x, z)(s), d(z, y)(t))$, whenever $s \geq \lambda_{1}(x, z), t \geq \lambda_{1}(z, y)$ and $s+t \geq \lambda_{1}(x, y)$.

If $d$ is a mapping from $X \times X$ into $G_{\infty}^{+}$and $(X, d, L, R)$ satisfies (FM-1)-(FM-3), then $(X, d, L, R)$ is called a generalized fuzzy metric space (briefly, GFMS).

From Lemma 2.1 and Definition 2.2, we obtain the following consequences.

Lemma 2.2 Let $(X, d, L, R)$ be a FMS, $[d(x, y)]_{\alpha}=\left[\lambda_{\alpha}(x, y), \rho_{\alpha}(x, y)\right]$ for $\alpha \in(0,1]$, where $x, y \in X$ are any two fixed elements. Then

(1) $\lim _{t \rightarrow-\infty} d(x, y)(t)=0=\lim _{t \rightarrow+\infty} d(x, y)(t)$;

(2) $d(x, y)(t)$ is a left-continuous and non-increasing function for $t \in\left(\lambda_{1}(x, y),+\infty\right)$;

(3) $\rho_{\alpha}(x, y)$ is a left-continuous and non-increasing function for $\alpha \in(0,1]$.

Lemma 2.3 (Xiao and Zhu [17]) Let $(X, d, L, R)$ be a FMS, and suppose that

(R-1) $R \leq \max$

(R-2) for each $\alpha \in(0,1]$, there exists $s \in(0, \alpha]$ such that $R(s, t)<\alpha$ for all $t \in(0, \alpha)$;

(R-3) $\lim _{a \rightarrow 0^{+}} R(a, a)=0$.

Then $(\mathrm{R}-1) \Rightarrow(\mathrm{R}-2) \Rightarrow(\mathrm{R}-3)$.

Remark 2.1 (Xiao et al. $[10,17]$ ) Since $R(t, s)=[\min (t, s)]^{1 / 2}$ satisfies (R-2) and does not satisfy (R-1), (R-2) does not imply (R-1). Since $R(t, s)=[\max (t, s)]^{1 / 2}$ satisfies (R-3) and does not satisfy (R-2), (R-3) does not imply (R-2).

Lemma 2.4 Let $(X, d, L, R)$ be a FMS. Then

(1) (R-1) $\Rightarrow$ for each $\alpha \in(0,1], \rho_{\alpha}(x, y) \leq \rho_{\alpha}(x, z)+\rho_{\alpha}(z, y)$ for all $x, y, z \in X$ (cf. [9]);

(2) (R-2) $\Rightarrow$ for each $\alpha \in(0,1]$, there exists $\mu=\mu(\alpha) \in(0, \alpha]$ such that $\rho_{\alpha}(x, y) \leq \rho_{\mu}(x, z)+\rho_{\alpha}(z, y)$ for all $x, y, z \in X$ (cf. [10]);

(3) (R-3) $\Rightarrow$ for each $\alpha \in(0,1]$, there exists $\mu=\mu(\alpha) \in(0, \alpha]$ such that $\rho_{\alpha}(x, y) \leq \rho_{\mu}(x, z)+\rho_{\mu}(z, y)$ for all $x, y, z \in X(c f .[16])$.

Lemma 2.5 (Kaleva and Seikkala [1]) Let $(X, d, L, R)$ be a FMS with (R-3). Then the family $\{U(\varepsilon, \alpha): \varepsilon>0, \alpha \in(0,1]\}$ of sets $U(\varepsilon, \alpha)=\left\{(x, y) \in X \times X: \rho_{\alpha}(x, y)<\varepsilon\right\}$ forms a basis for a Hausdorff uniformity on $X \times X$. Moreover, the sets

$$
N_{x}(\varepsilon, \alpha)=\left\{y \in X: \rho_{\alpha}(x, y)<\varepsilon\right\}
$$

form a basis for a Hausdorff topology on $X$ and this topology is metrizable.

According to Lemma 2.5, convergence in a FMS $(X, d, L, R)$ can be defined by sequences.

Definition 2.3 (Kaleva and Seikkala [1]) Let $(X, d, L, R)$ be a FMS. 
(1) A sequence $\left\{x_{n}\right\}$ in $X$ is said to be convergent to $x$ (we write $x_{n} \rightarrow x$ or $\left.\lim _{n \rightarrow \infty} x_{n}=x\right)$ if $\lim _{n \rightarrow \infty} d\left(x_{n}, x\right)=\overline{0}$, i.e., $\lim _{n \rightarrow \infty} \rho_{\alpha}\left(x_{n}, x\right)=0$ for all $\alpha \in(0,1]$;

(2) A sequence $\left\{x_{n}\right\}$ in $X$ is said to be a Cauchy sequence in $X$ if $\lim _{m, n \rightarrow \infty} d\left(x_{m}, x_{n}\right)=\overline{0}$, equivalently, for any given $\varepsilon>0$ and $\alpha \in(0,1]$, there exists $N=N(\varepsilon, \alpha) \in \mathbb{Z}^{+}$such that $\rho_{\alpha}\left(x_{m}, x_{n}\right)<\varepsilon$, whenever $m, n \geq N$;

(3) $(X, d, L, R)$ is said to be complete if each Cauchy sequence in $X$ is convergent to some point in $X$.

Lemma 2.6 (Xiao et al. [10]) Let $(X, d, L, R)$ be a FMS with (R-2). Then, for each $\alpha \in(0,1]$, $\rho_{\alpha}(x, y)$ is continuous at $(x, y) \in X \times X$.

Definition 2.4 Let $\varphi: \mathbb{R}^{+} \rightarrow \mathbb{R}^{+}$be a function and let $\varphi^{n}(t)$ denote the $n$th iteration of $\varphi(t)$.

(1) $\varphi$ is said to satisfy condition $(\Phi)$ if it is nondecreasing, right-continuous and $\varphi(t)<t$ for all $t>0$.

(2) $\varphi$ is said to satisfy condition $\left(\Phi_{0}\right)$ if it is nondecreasing, right-continuous and $\sum_{n=0}^{\infty} \varphi^{n}(t)<+\infty$ for all $t>0$.

Remark 2.2 Obviously, if $\varphi(t)$ satisfies condition $\left(\Phi_{0}\right)$, then $\varphi(t)<t$ for all $t>0$, i.e., $\left(\Phi_{0}\right) \subset$ $(\Phi)$. Since $\varphi(t)=\frac{t}{1+t}$ satisfies condition $(\Phi)$ and does not satisfy condition $\left(\Phi_{0}\right),\left(\Phi_{0}\right)$ does not imply $(\Phi)$, i.e., $(\Phi) \not \subset\left(\Phi_{0}\right)$. Also, if $\varphi(t)$ satisfies condition $(\Phi)$, then $\varphi(0)=0$.

Definition 2.5 A function $F: \mathbb{R}_{4}^{+} \rightarrow \mathbb{R}$ is called a real function satisfying an implicit relation if the following conditions are satisfied:

(A-1) $F$ is right-lower semi-continuous;

(A-2) There exists $\varphi \in\left(\Phi_{0}\right)$ or $(\Phi)$ such that $F(u, v, 0, u) \leq 0$ or $F(u, v, u, 0) \leq 0$ for $u, v \in \mathbb{R}^{+}$implies $u \leq \varphi(v)$.

We denote by $\mathcal{A}_{\varphi}$ the collection of all real functions $F: \mathbb{R}_{4}^{+} \rightarrow \mathbb{R}$ satisfying an implicit relation.

The following examples show that the $\mathcal{A}_{\varphi}$ is a largish class of real functions.

Example 1 Let $\varphi \in\left(\Phi_{0}\right)$ or $(\Phi)$. We define the functions $F_{1}, F_{2}: \mathbb{R}_{4}^{+} \rightarrow \mathbb{R}$ as follows:

$$
\begin{aligned}
& F_{1}\left(t_{1}, t_{2}, t_{3}, t_{4}\right)=t_{1}-\varphi\left(\max \left\{t_{2}, t_{3}, t_{4}\right\}\right), \\
& F_{2}\left(t_{1}, t_{2}, t_{3}, t_{4}\right)=t_{1}-\max \left\{\varphi\left(t_{2}\right), \varphi\left(t_{3}\right), \varphi\left(t_{4}\right)\right\},
\end{aligned}
$$

respectively. Then $F_{1}, F_{2} \in \mathcal{A}_{\varphi}$.

In fact, since $\varphi \in\left(\Phi_{0}\right)$ or $(\Phi)$, by the right-continuity of $\varphi$, we know that $F_{1}$ satisfies condition (A-1). If $F_{1}(u, v, 0, u) \leq 0$ or $F_{1}(u, v, u, 0) \leq 0$, then we have $u \leq \varphi(\max \{u, v\})<$ $\max \{u, v\}$ for $u \neq v$. This implies $u<v$, and so $u \leq \varphi(\max \{u, v\})=\varphi(v)$. In addition, if $u=v$, then $u=0 \leq \varphi(v)=\varphi(0)=0$. This shows that $F_{1}$ satisfies condition (A-2). Hence $F_{1} \in \mathcal{A}_{\varphi}$. 
Example 2 Let $\varphi \in\left(\Phi_{0}\right)$ or $(\Phi)$. We define the functions $F_{3}, F_{4}: \mathbb{R}_{4}^{+} \rightarrow \mathbb{R}$, respectively, as follows:

$$
\begin{aligned}
& F_{3}\left(t_{1}, t_{2}, t_{3}, t_{4}\right)=t_{1}-\varphi\left(\lambda_{1} t_{2}+\lambda_{2} t_{3}+\lambda_{3} t_{4}\right), \\
& F_{4}\left(t_{1}, t_{2}, t_{3}, t_{4}\right)=t_{1}-\left(\lambda_{1} \varphi\left(t_{2}\right)+\lambda_{2} \varphi\left(t_{3}\right)+\lambda_{3} \varphi\left(t_{4}\right)\right),
\end{aligned}
$$

where $\lambda_{1}, \lambda_{2}, \lambda_{3} \geq 0$ and $\lambda_{1}+\lambda_{2}+\lambda_{3}=1$. Then $F_{3}, F_{4} \in \mathcal{A}_{\varphi}$.

Obviously, $F_{3}$ satisfies condition (A-1).

Now suppose that $F_{3}(u, v, 0, u) \leq 0$ or $F_{3}(u, v, u, 0) \leq 0$. If $u=0$, then $u \leq \varphi(v)$ holds evidently. If $u>0$, then we have $\varphi(u)<u \leq \varphi\left(\lambda_{1} v+\lambda_{3} u\right)$ or $\varphi(u)<u \leq \varphi\left(\lambda_{1} v+\lambda_{2} u\right)$, which implies that $u<\lambda_{1} v+\lambda_{3} u$ or $u<\lambda_{1} v+\lambda_{2} u$, and so $u<v$. Hence, we have $u \leq \varphi\left(\lambda_{1} v+\lambda_{3} u\right) \leq$ $\varphi(v)$ or $u \leq \varphi\left(\lambda_{1} v+\lambda_{2} u\right) \leq \varphi(v)$, i.e., (A-2) holds. Therefore $F_{3} \in \mathcal{A}_{\varphi}$.

Similarly, it is easy to prove that $F_{4} \in \mathcal{A}_{\varphi}$.

Example 3 Let $\varphi \in\left(\Phi_{0}\right)$ or $(\Phi)$. The function $F_{5}: \mathbb{R}_{4}^{+} \rightarrow \mathbb{R}$ is defined by

$$
F_{5}\left(t_{1}, t_{2}, t_{3}, t_{4}\right)=t_{1}-\varphi\left(\frac{t_{1} t_{2}+t_{3} t_{4}}{t_{2}+t_{3}+t_{4}+1}\right),
$$

then $F_{5} \in \mathcal{A}_{\varphi}$.

In fact, it is easy to see that $F_{5}$ satisfies condition (A-1). Now suppose that $F_{5}(u, v, 0, u) \leq$ 0 or $F_{5}(u, v, u, 0) \leq 0$. If $u=0$, then $u \leq \varphi(v)$ holds evidently. If $u>0$, then we have $u \leq$ $\varphi\left(\frac{u v}{u+v+1}\right)$, which implies that $u \leq \varphi(v)$, i.e., (A-2) holds. Therefore $F_{5} \in \mathcal{A}_{\varphi}$.

Example 4 Let $\varphi \in\left(\Phi_{0}\right)$ or $(\Phi)$. The function $F_{6}: \mathbb{R}_{4}^{+} \rightarrow \mathbb{R}$ is defined by

$$
F_{6}\left(t_{1}, t_{2}, t_{3}, t_{4}\right)=\left(1+t_{2}\right) t_{1}-\varphi\left(\max \left\{t_{1} t_{2}, t_{3} t_{4}\right\}\right)-\varphi\left(\max \left\{t_{2}, t_{3}, t_{4}\right\}\right),
$$

then $F_{6} \in \mathcal{A}_{\varphi}$.

In fact, it is easy to see that $F_{6}$ satisfies condition (A-1). Now suppose that $F_{6}(u, v, 0, u) \leq$ 0 or $F_{6}(u, v, u, 0) \leq 0$. If $u=0$, then $u \leq \varphi(v)$ holds evidently. If $u>0$, then we have $(1+v) u \leq$ $\varphi(u v)+\varphi(\max \{u, v\})<u v+\max \{u, v\}$, which implies that $u<\max \{u, v\}$, and so $u<v$. Hence, we have $(1+v) u \leq \varphi(u v)+\varphi(v)<u v+\varphi(v) \Rightarrow u<\varphi(v)$, i.e., (A-2) holds. Therefore $F_{6} \in \mathcal{A}_{\varphi}$.

Definition 2.6 (Aliouche and Fisher [13]) Let $f: \mathbb{R}_{4}^{+} \rightarrow \mathbb{R}$ be a function satisfying the following conditions:

(F-1) $f$ is lower semi-continuous;

(F-2) There exists $0<c<1$ such that $f(u, v, 0, u) \leq 0$ or $f(u, v, u, 0) \leq 0$ for $u, v \in \mathbb{R}^{+}$ implies $u<c v$.

We denote by $\mathcal{F}$ the collection of all real functions $f: \mathbb{R}_{4}^{+} \rightarrow \mathbb{R}$ satisfying the conditions of Definition 2.6.

Remark 2.3 Taking $\varphi(t)=c t, c \in(0,1), t \geq 0$, we have $\varphi \in\left(\Phi_{0}\right) \subset(\Phi)$. Then it is easy to see that $\mathcal{F} \subset \mathcal{A}_{\varphi}$, which implies that the implicit relation of Definition 2.5 is a generalization of Aliouche and Fisher [13, implicit relation]. 


\section{Main results}

Theorem 3.1 Let $(X, d, L, R)$ and $(Y, \widetilde{d}, \widetilde{L}, \widetilde{R})$ be two complete FMS $s$ with $R$ and $\widetilde{R}$ satisfying (R-2). Let $T: X \rightarrow Y$ and $S: Y \rightarrow X$ be two non-self mappings, and $\varphi_{1}, \varphi_{2} \in\left(\Phi_{0}\right)$. If there exist $F \in \mathcal{A}_{\varphi_{1}}$ and $G \in \mathcal{A}_{\varphi_{2}}$ such that

$$
\begin{aligned}
& F\left(\tilde{\rho}_{\alpha}(T x, T S y), \rho_{\alpha}(x, S y), \tilde{\rho}_{\alpha}(y, T x), \tilde{\rho}_{\alpha}(y, T S y)\right) \leq 0, \\
& G\left(\rho_{\alpha}(S y, S T x), \tilde{\rho}_{\alpha}(y, T x), \rho_{\alpha}(x, S y), \rho_{\alpha}(x, S T x)\right) \leq 0
\end{aligned}
$$

for all $x \in X, y \in Y$ and $\alpha \in(0,1]$, then ST has a unique fixed point $x_{*} \in X$, and TS has a unique fixed point $y_{*} \in Y$ with $T x_{*}=y_{*}, S y_{*}=x_{*}$.

Proof For any given $x_{0} \in X$, we construct a sequence $\left\{x_{n}\right\}_{n=0}^{\infty}$ in $X$ and a sequence $\left\{y_{n}\right\}_{n=1}^{\infty}$ in $Y$, respectively, as follows:

$$
x_{n}=(S T)^{n} x_{0}, \quad y_{n}=T(S T)^{n-1} x_{0} \quad \text { for all } n=1,2,3, \ldots
$$

Obviously, we have $y_{n}=T x_{n-1}, S y_{n}=x_{n}, T S y_{n}=T x_{n}=y_{n+1}$ for all $n=1,2,3, \ldots$

For $y_{n}, x_{n-1}$, applying (3.1), we obtain for each $\alpha \in(0,1]$

$$
\begin{gathered}
F\left(\tilde{\rho}_{\alpha}\left(T x_{n-1}, T S y_{n}\right), \rho_{\alpha}\left(x_{n-1}, S y_{n}\right), \tilde{\rho}_{\alpha}\left(y_{n}, T x_{n-1}\right), \tilde{\rho}_{\alpha}\left(y_{n}, T S y_{n}\right)\right) \\
\quad=F\left(\widetilde{\rho}_{\alpha}\left(y_{n}, y_{n+1}\right), \rho_{\alpha}\left(x_{n-1}, x_{n}\right), 0, \tilde{\rho}_{\alpha}\left(y_{n}, y_{n+1}\right)\right) \leq 0 .
\end{gathered}
$$

Note that $F \in \mathcal{A}_{\varphi_{1}}$, it is not difficult to see that

$$
\tilde{\rho}_{\alpha}\left(y_{n}, y_{n+1}\right) \leq \varphi_{1}\left(\rho_{\alpha}\left(x_{n-1}, x_{n}\right)\right) \quad \text { for each } \alpha \in(0,1]
$$

Again, for $y_{n}, x_{n}$, applying (3.2), we obtain for each $\alpha \in(0,1]$

$$
\begin{gathered}
G\left(\rho_{\alpha}\left(S y_{n}, S T x_{n}\right), \tilde{\rho}_{\alpha}\left(y_{n}, T x_{n}\right), \rho_{\alpha}\left(x_{n}, S y_{n}\right), \rho_{\alpha}\left(x_{n}, S T x_{n}\right)\right) \\
=G\left(\rho_{\alpha}\left(x_{n}, x_{n+1}\right), \tilde{\rho}_{\alpha}\left(y_{n}, y_{n+1}\right), 0, \rho_{\alpha}\left(x_{n}, x_{n+1}\right)\right) \leq 0 .
\end{gathered}
$$

Note that $G \in \mathcal{A}_{\varphi_{2}}$, we have

$$
\rho_{\alpha}\left(x_{n}, x_{n+1}\right) \leq \varphi_{2}\left(\tilde{\rho}_{\alpha}\left(y_{n}, y_{n+1}\right)\right) \quad \text { for each } \alpha \in(0,1]
$$

Since $\varphi_{1}, \varphi_{2} \in\left(\Phi_{0}\right)$, by Remark 2.2, (3.3) and (3.4), we can obtain

$$
\rho_{\alpha}\left(x_{n}, x_{n+1}\right) \leq \varphi_{2}\left(\varphi_{1}\left(\rho_{\alpha}\left(x_{n-1}, x_{n}\right)\right)\right) \leq \varphi_{1}\left(\rho_{\alpha}\left(x_{n-1}, x_{n}\right)\right) \quad \text { for each } \alpha \in(0,1] \text {, }
$$

and

$$
\widetilde{\rho}_{\alpha}\left(y_{n}, y_{n+1}\right) \leq \varphi_{1}\left(\varphi_{2}\left(\widetilde{\rho}_{\alpha}\left(y_{n-1}, y_{n}\right)\right)\right) \leq \varphi_{2}\left(\widetilde{\rho}_{\alpha}\left(y_{n-1}, y_{n}\right)\right) \quad \text { for each } \alpha \in(0,1] \text {. }
$$

Using the inductive method, for $n=1,2,3, \ldots$, we have

$$
\rho_{\alpha}\left(x_{n}, x_{n+1}\right) \leq \varphi_{1}^{n}\left(\rho_{\alpha}\left(x_{0}, x_{1}\right)\right) \quad \text { for each } \alpha \in(0,1]
$$


and

$$
\tilde{\rho}_{\alpha}\left(y_{n}, y_{n+1}\right) \leq \varphi_{2}^{n-1}\left(\widetilde{\rho}_{\alpha}\left(y_{1}, y_{2}\right)\right) \text { for each } \alpha \in(0,1]
$$

In the next step, we show that $\left\{x_{n}\right\}$ is a Cauchy sequence in $X$. Since $(X, d, L, R)$ is with (R-2), it follows from Lemma 2.4(2) that for each $\alpha \in(0,1]$, there exists $\mu=\mu(\alpha) \in(0, \alpha]$ such that

$$
\rho_{\alpha}(x, y) \leq \rho_{\mu}(x, z)+\rho_{\alpha}(z, y) \quad \text { for all } x, y, z \in X
$$

For $m, n \in \mathbb{N}$ and $n<m$, by (3.7), (3.5) and Lemma 2.2(3), we have

$$
\begin{aligned}
\rho_{\alpha}\left(x_{n}, x_{m}\right) & \leq \rho_{\mu}\left(x_{n}, x_{n+1}\right)+\rho_{\alpha}\left(x_{n+1}, x_{m}\right) \\
& \leq \rho_{\mu}\left(x_{n}, x_{n+1}\right)+\rho_{\mu}\left(x_{n+1}, x_{n+2}\right)+\cdots+\rho_{\mu}\left(x_{m-2}, x_{m-1}\right)+\rho_{\alpha}\left(x_{m-1}, x_{m}\right) \\
& \leq \rho_{\mu}\left(x_{n}, x_{n+1}\right)+\rho_{\mu}\left(x_{n+1}, x_{n+2}\right)+\cdots+\rho_{\mu}\left(x_{m-2}, x_{m-1}\right)+\rho_{\mu}\left(x_{m-1}, x_{m}\right) \\
& \leq \sum_{i=n}^{m-1} \varphi_{1}^{i}\left(\rho_{\mu}\left(x_{0}, x_{1}\right)\right) \leq \sum_{i=n}^{\infty} \varphi_{1}^{i}\left(\rho_{\mu}\left(x_{0}, x_{1}\right)\right) .
\end{aligned}
$$

Since $\varphi_{1} \in\left(\Phi_{0}\right)$, i.e., $\sum_{n=1}^{\infty} \varphi_{1}^{n}(t)<+\infty$ for all $t>0$, it follows from (3.8) that $\left\{x_{n}\right\}$ is a Cauchy sequence in $X$. Hence, by the completeness of $(X, d, L, R)$, there exists $x_{*} \in X$ such that $\lim _{n \rightarrow \infty} x_{n}=x_{*}$. By the similar reasoning process, from (3.6), (3.7), $\varphi_{2} \in\left(\Phi_{0}\right)$ and Lemma 2.2(3), we can prove that $\left\{y_{n}\right\}$ is a Cauchy sequence in $Y$. Hence, by the completeness of $(Y, \widetilde{d}, \widetilde{L}, \widetilde{R})$, there exists $y_{*} \in Y$ such that $\lim _{n \rightarrow \infty} y_{n}=y_{*}$.

Now we prove that $x_{*}$ is a fixed point of $S T$ and $y_{*}$ is a fixed point of TS. For $x_{*}, y_{n-1}$, applying (3.1), we have for each $\alpha \in(0,1]$

$$
\begin{gathered}
F\left(\widetilde{\rho}_{\alpha}\left(T x_{*}, T S y_{n-1}\right), \rho_{\alpha}\left(x_{*}, S y_{n-1}\right), \tilde{\rho}_{\alpha}\left(y_{n-1}, T x_{*}\right), \widetilde{\rho}_{\alpha}\left(y_{n-1}, T S y_{n-1}\right)\right) \\
\quad=F\left(\widetilde{\rho}_{\alpha}\left(T x_{*}, y_{n}\right), \rho_{\alpha}\left(x_{*}, x_{n-1}\right), \widetilde{\rho}_{\alpha}\left(y_{n-1}, T x_{*}\right), \tilde{\rho}_{\alpha}\left(y_{n-1}, y_{n}\right)\right) \leq 0 .
\end{gathered}
$$

Let $n \rightarrow \infty$, by the lower semi-continuity of $F$ and Lemma 2.6, we have for each $\alpha \in(0,1]$

$$
\begin{aligned}
& F\left(\tilde{\rho}_{\alpha}\left(T x_{*}, y_{*}\right), 0, \tilde{\rho}_{\alpha}\left(y_{*}, T x_{*}\right), 0\right) \\
& \quad \leq \liminf _{n \rightarrow \infty} F\left(\widetilde{\rho}_{\alpha}\left(T x_{*}, y_{n}\right), \rho_{\alpha}\left(x_{*}, x_{n-1}\right), \widetilde{\rho}_{\alpha}\left(y_{n-1}, T x_{*}\right), \widetilde{\rho}_{\alpha}\left(y_{n-1}, y_{n}\right)\right) \leq 0 .
\end{aligned}
$$

Note that $F \in \mathcal{A}_{\varphi_{1}}$ and (A-2) of Definition 2.5, we can obtain $\widetilde{\rho}_{\alpha}\left(T x_{*}, y_{*}\right) \leq \varphi_{1}(0)=0$ for each $\alpha \in(0,1]$, i.e., $T x_{*}=y_{*}$. Similarly, we can prove that $S y_{*}=x_{*}$. Hence, $S T x_{*}=S y_{*}=x_{*}$ and $T S y_{*}=T x_{*}=y_{*}$, which imply that $x_{*}$ is a fixed point of $S T$ and $y_{*}$ is a fixed point of TS.

Finally, we show the uniqueness of a fixed point. If $y^{*}$ is another fixed point of $T S$, then by (3.1) we have for each $\alpha \in(0,1]$

$$
\begin{gathered}
F\left(\widetilde{\rho}_{\alpha}\left(T x_{*}, T S y^{*}\right), \rho_{\alpha}\left(x_{*}, S y^{*}\right), \widetilde{\rho}_{\alpha}\left(y^{*}, T x_{*}\right), \widetilde{\rho}_{\alpha}\left(y^{*}, T S y^{*}\right)\right) \\
\quad=F\left(\widetilde{\rho}_{\alpha}\left(y_{*}, y^{*}\right), \rho_{\alpha}\left(S y_{*}, S y^{*}\right), \widetilde{\rho}_{\alpha}\left(y^{*}, y_{*}\right), 0\right) \leq 0 .
\end{gathered}
$$

Note that $F \in \mathcal{A}_{\varphi_{1}}$, it is not difficult to obtain that $\tilde{\rho}_{\alpha}\left(y_{*}, y^{*}\right) \leq \varphi_{1}\left(\rho_{\alpha}\left(S y_{*}, S y^{*}\right)\right)$ for each 
$\alpha \in(0,1]$. We claim that $S y_{*}=S y^{*}$. In fact, if $S y_{*} \neq S y^{*}$, there exists $\alpha_{0} \in(0,1]$ such that $\rho_{\alpha_{0}}\left(S y_{*}, S y^{*}\right)>0$. From (3.2), it follows that

$$
\begin{gathered}
G\left(\rho_{\alpha_{0}}\left(S y^{*}, S T x_{*}\right), \tilde{\rho}_{\alpha_{0}}\left(y^{*}, T x_{*}\right), \rho_{\alpha_{0}}\left(x_{*}, S y^{*}\right), \rho_{\alpha_{0}}\left(x_{*}, S T x_{*}\right)\right) \\
=G\left(\rho_{\alpha_{0}}\left(S y^{*}, S y_{*}\right), \tilde{\rho}_{\alpha_{0}}\left(y^{*}, y_{*}\right), \rho_{\alpha_{0}}\left(S y_{*}, S y^{*}\right), 0\right) \leq 0 .
\end{gathered}
$$

Note that $G \in \mathcal{A}_{\varphi_{2}}$, we have $\rho_{\alpha_{0}}\left(S y^{*}, S y_{*}\right) \leq \varphi_{2}\left(\widetilde{\rho}_{\alpha_{0}}\left(y^{*}, y_{*}\right)\right)$. Since $\varphi_{1}, \varphi_{2} \in\left(\Phi_{0}\right)$, by Remark 2.2, it is not difficult to obtain that

$$
\tilde{\rho}_{\alpha_{0}}\left(y_{*}, y^{*}\right) \leq \varphi_{1}\left(\rho_{\alpha_{0}}\left(S y_{*}, S y^{*}\right)\right)<\rho_{\alpha_{0}}\left(S y_{*}, S y^{*}\right) \leq \varphi_{2}\left(\tilde{\rho}_{\alpha_{0}}\left(y^{*}, y_{*}\right)\right) \leq \tilde{\rho}_{\alpha_{0}}\left(y^{*}, y_{*}\right)
$$

which is a contradiction. Hence, $S y_{*}=S y^{*}$, i.e., $\widetilde{\rho}_{\alpha}\left(y_{*}, y^{*}\right) \leq \varphi_{1}\left(\rho_{\alpha}\left(S y_{*}, S y^{*}\right)\right)=\varphi_{1}(0)=0$ for each $\alpha \in(0,1]$. This shows that $y_{*}=y^{*}$, i.e., the uniqueness of a fixed point for TS is true. Similarly, we can prove the uniqueness of a fixed point for $S T$. So, the proof of Theorem 3.1 is finished.

Theorem 3.2 Let $(X, d, L, R)$ and $(Y, \widetilde{d}, \widetilde{L}, \widetilde{R})$ be two complete FMS $s$ with $R$ and $\widetilde{R}$ satisfying (R-2). Let $T: X \rightarrow Y$ and $S: Y \rightarrow X$ be two non-self mappings, and $\varphi_{1}, \varphi_{2} \in\left(\Phi_{0}\right)$. If there exist $F \in \mathcal{A}_{\varphi_{1}}$ and $G \in \mathcal{A}_{\varphi_{2}}$ such that $u-F(u, v, w, s) \geq \tilde{\lambda}_{1}(T x, T S y), u-G(u, v, w, s) \geq$ $\lambda_{1}(S y, S T x)$ and

$$
\begin{aligned}
& \tilde{d}(T x, T S y)(u-F(u, v, w, s)) \\
& \quad \leq \max \{\tilde{d}(T x, T S y)(u), d(x, S y)(v), \tilde{d}(y, T x)(w), \tilde{d}(y, T S y)(s)\}, \\
& d(S y, S T x)(u-G(u, v, w, s)) \\
& \quad \leq \max \{d(S y, S T x)(u), \tilde{d}(y, T x)(v), d(x, S y)(w), d(x, S T x)(s)\}
\end{aligned}
$$

for all $x \in X, y \in Y$ and $u, v, w, s \geq 0$, then ST has a unique fixed point $x_{*} \in X$, and TS has a unique fixed point $y_{*} \in Y$ with $T x_{*}=y_{*}, S y_{*}=x_{*}$.

Proof Now, we use inequality (3.9) to prove that inequality (3.1) holds. In fact, for each $x \in X, y \in Y$ and $\alpha \in(0,1]$, if we set $\widetilde{\rho}_{\alpha}(T x, T S y)=u, \rho_{\alpha}(x, S y)=v, \widetilde{\rho}_{\alpha}(y, T x)=w$, $\tilde{\rho}_{\alpha}(y, T S y)=s$, then for any $\varepsilon>0$, it is obvious that $\widetilde{d}(T x, T S y)(u+\varepsilon)<\alpha, d(x, S y)(v+\varepsilon)<\alpha$, $\widetilde{d}(y, T x)(w+\varepsilon)<\alpha, \widetilde{d}(y, T S y)(s+\varepsilon)<\alpha$. By (3.9), we have $\widetilde{d}(T x, T S y)(u+\varepsilon-F(u+\varepsilon, v+\varepsilon, w+$ $\varepsilon, s+\varepsilon))<\alpha$, which implies that

$$
\tilde{\rho}_{\alpha}(T x, T S y)=u<u+\varepsilon-F(u+\varepsilon, v+\varepsilon, w+\varepsilon, s+\varepsilon),
$$

i.e.,

$$
F\left(\widetilde{\rho}_{\alpha}(T x, T S y)+\varepsilon, \rho_{\alpha}(x, S y)+\varepsilon, \tilde{\rho}_{\alpha}(y, T x)+\varepsilon, \tilde{\rho}_{\alpha}(y, T S y)+\varepsilon\right)<\varepsilon
$$

Then, by the arbitrariness of $\varepsilon$ and the right-lower semi-continuity of $F$, we have

$$
\begin{aligned}
& F\left(\widetilde{\rho}_{\alpha}(T x, T S y), \rho_{\alpha}(x, S y), \tilde{\rho}_{\alpha}(y, T x), \widetilde{\rho}_{\alpha}(y, T S y)\right) \\
& \quad \leq \liminf _{\varepsilon \rightarrow 0^{+}} F\left(\widetilde{\rho}_{\alpha}(T x, T S y)+\varepsilon, \rho_{\alpha}(x, S y)+\varepsilon, \widetilde{\rho}_{\alpha}(y, T x)+\varepsilon, \widetilde{\rho}_{\alpha}(y, T S y)+\varepsilon\right) \\
& \quad \leq 0
\end{aligned}
$$


for each $x \in X, y \in Y$ and $\alpha \in(0,1]$, i.e., inequality (3.1) holds for all $x, y \in X$ and $\alpha \in$ $(0,1]$.

Similarly, by inequality (3.10), we can prove that inequality (3.2) also holds. Moreover, the other conditions in Theorem 3.1 are satisfied, thus by Theorem 3.1, the theorem is proved.

In Theorem 3.2, taking $(X, d, L, R)=(Y, \widetilde{d}, \widetilde{L}, \widetilde{R}), S=T$, we obtain the following corollary.

Corollary 3.1 Let $(X, d, L, R)$ be a complete FMS with $R$ satisfying (R-2). Let $T: X \rightarrow X$ be a self-mapping, and $\varphi \in\left(\Phi_{0}\right)$. If there exists $F \in \mathcal{A}_{\varphi}$ such that $u-F(u, v, w, s) \geq \lambda_{1}\left(T x, T^{2} y\right)$ and

$$
\begin{aligned}
& d\left(T x, T^{2} y\right)(u-F(u, v, w, s)) \\
& \quad \leq \max \left\{d\left(T x, T^{2} y\right)(u), d(x, T y)(v), d(y, T x)(w), d\left(y, T^{2} y\right)(s)\right\}
\end{aligned}
$$

for all $x, y \in X$ and $u, v, w, s \geq 0$, then $T$ has a unique fixed point in $X$.

Corollary 3.2 Let $(X, d, L, R)$ be a complete FMS with $R$ satisfying $(\mathrm{R}-2)$, and let $T: X \rightarrow X$ be a self-mapping. If there exists $\varphi \in\left(\Phi_{0}\right)$ such that $\varphi(v) \geq \lambda_{1}(T x, T y)$ and

$$
d(T x, T y)(\varphi(v)) \leq d(x, y)(v)
$$

for all $x \in X, y \in T(X)$ and $v \geq 0$, then $T$ has a unique fixed point in $X$.

Proof Taking $F\left(t_{1}, t_{2}, t_{3}, t_{4}\right)=t_{1}-\varphi\left(\lambda_{1} t_{2}+\lambda_{2} t_{3}+\lambda_{3} t_{4}\right), \lambda_{1}=1, \lambda_{2}=\lambda_{3}=0$, from Example 2, we obtain $F=F_{3} \in \mathcal{A}_{\varphi}$. Furthermore, for all $x, y \in X$ and $u, v, w, s \geq 0$, by $T y \in T(X)$ and (3.12), we have

$$
\begin{aligned}
d\left(T x, T^{2} y\right)(\varphi(v)) & =d\left(T x, T^{2} y\right)(u-(u-\varphi(v))) \leq d(x, T y)(v) \\
& \leq \max \left\{d\left(T x, T^{2} y\right)(u), d(x, T y)(v), d(y, T x)(w), d\left(y, T^{2} y\right)(s)\right\},
\end{aligned}
$$

which implies that (3.11) holds. Therefore, the conclusion follows from Corollary $3.1 \mathrm{im}$ mediately.

Remark 3.1 Corollary 3.2 is a fuzzy version of the Boyd-Wong-type nonlinear contraction theorem (see [18]).

Theorem 3.3 Let $(X, d, L, R)$ and $(Y, \widetilde{d}, \widetilde{L}, \widetilde{R})$ be two complete FMS $s$ with $R$ and $\widetilde{R}$ satisfying (R-2). Let $\varphi_{1}, \varphi_{2} \in(\Phi)$. Suppose that $T: X \rightarrow Y$ and $S: Y \rightarrow X$ are two continuous non-self mappings satisfying the following conditions:

(1) There exists $F \in \mathcal{A}_{\varphi_{1}}$ such that

$$
F\left(\widetilde{\rho}_{\alpha}(T x, T S y), \rho_{\alpha}(x, S y), \tilde{\rho}_{\alpha}(y, T x), \tilde{\rho}_{\alpha}(y, T S y)\right) \leq 0
$$

for all $x \in X, y \in Y$ and $\alpha \in(0,1]$ with $x \neq S y$;

(2) There exists $G \in \mathcal{A}_{\varphi_{2}}$ such that

$$
G\left(\rho_{\alpha}(S y, S T x), \tilde{\rho}_{\alpha}(y, T x), \rho_{\alpha}(x, S y), \rho_{\alpha}(x, S T x)\right) \leq 0
$$

for all $x \in X, y \in Y$ and $\alpha \in(0,1]$ with $y \neq T x$; 
(3) There exists $x_{0} \in X$ such that $\left\{(S T)^{n} x_{0}\right\}$ has an accumulation point in $X$.

Then ST has a unique fixed point $x_{*} \in X$, and TS has a unique fixed point $y_{*} \in Y$ with $T x_{*}=y_{*}, S y_{*}=x_{*}$.

Proof From condition (3), we can construct a sequence $\left\{x_{n}\right\}_{n=0}^{\infty}$ in $X$ and a sequence $\left\{y_{n}\right\}_{n=1}^{\infty}$ in $Y$, respectively, as follows:

$$
x_{n}=(S T)^{n} x_{0}, \quad y_{n}=T(S T)^{n-1} x_{0}, \quad \text { for all } n=1,2,3, \ldots
$$

Obviously, we have $y_{n}=T x_{n-1}, S y_{n}=x_{n}, T S y_{n}=T x_{n}=y_{n+1}$ for all $n=1,2,3, \ldots$ If $x_{n_{0}}=x_{n_{0}+1}$ for some $n_{0}$, then $x_{*}=x_{n_{0}}$ is a fixed point of ST. So, we can assume that $x_{n} \neq x_{n+1}$ for all $n=0,1,2, \ldots$. Then, for all $n=1,2,3, \ldots$, it is obvious that $y_{n} \neq y_{n+1}$.

Again by condition (3), we can assume that $z \in X$ is an accumulation point of $\left\{(S T)^{n} x_{0}\right\}=$ $\left\{x_{n}\right\}$. Then there exists a subsequence $\left\{x_{n_{i}}\right\}$ of $\left\{x_{n}\right\}$ such that $\lim _{i \rightarrow \infty} x_{n_{i}}=z$. Let $T z=y_{*}$. Next we show that $y_{*}$ is a fixed point of TS.

If $T S y_{*} \neq y_{*}$, then by $T z=y_{*}$, we have $T S T z \neq T z$, which implies that $S T z \neq z$, i.e., $z \neq S y_{*}$. It follows from (3.13) that for all $\alpha \in(0,1]$,

$$
\begin{aligned}
& F\left(\tilde{\rho}_{\alpha}\left(T z, T S y_{*}\right), \rho_{\alpha}\left(z, S y_{*}\right), \tilde{\rho}_{\alpha}\left(y_{*}, T z\right), \tilde{\rho}_{\alpha}\left(y_{*}, T S y_{*}\right)\right) \\
& \quad=F\left(\widetilde{\rho}_{\alpha}\left(T z, T S y_{*}\right), \rho_{\alpha}\left(z, S y_{*}\right), 0, \tilde{\rho}_{\alpha}\left(T z, T S y_{*}\right)\right) \leq 0 .
\end{aligned}
$$

Note that $F \in \mathcal{A}_{\varphi_{1}}$, it is not difficult to see that for all $\alpha \in(0,1]$,

$$
\tilde{\rho}_{\alpha}\left(T z, T S y_{*}\right) \leq \varphi_{1}\left(\rho_{\alpha}\left(z, S y_{*}\right)\right) \quad \Leftrightarrow \quad \tilde{\rho}_{\alpha}(T z, T S T z) \leq \varphi_{1}\left(\rho_{\alpha}(z, S T z)\right) .
$$

Similarly, by $T S y_{*} \neq y_{*}$ and (3.14), we obtain for all $\alpha \in(0,1]$

$$
\begin{gathered}
G\left(\rho_{\alpha}\left(S y_{*}, S T S y_{*}\right), \tilde{\rho}_{\alpha}\left(y_{*}, T S y_{*}\right), \rho_{\alpha}\left(S y_{*}, S y_{*}\right), \rho_{\alpha}\left(S y_{*}, S T S y_{*}\right)\right) \\
=G\left(\rho_{\alpha}\left(S y_{*}, S T S y_{*}\right), \tilde{\rho}_{\alpha}\left(y_{*}, T S y_{*}\right), 0, \rho_{\alpha}\left(S y_{*}, S T S y_{*}\right)\right) \leq 0 .
\end{gathered}
$$

Note that $G \in \mathcal{A}_{\varphi_{2}}$, we have for all $\alpha \in(0,1]$

$$
\begin{aligned}
& \rho_{\alpha}\left(S y_{*}, S T S y_{*}\right) \leq \varphi_{2}\left(\widetilde{\rho}_{\alpha}\left(y_{*}, T S y_{*}\right)\right) \\
& \quad \Leftrightarrow \quad \rho_{\alpha}(S T z, S T S T z) \leq \varphi_{2}\left(\widetilde{\rho}_{\alpha}(T z, T S T z)\right) .
\end{aligned}
$$

Since $S T z \neq z$, we know that there exists $\alpha_{0} \in(0,1]$ such that $\rho_{\alpha_{0}}(S T z, z)>0$. From $\varphi_{1}, \varphi_{2} \in(\Phi)$, (3.15) and (3.16), we can obtain

$$
\rho_{\alpha_{0}}\left(S T z,(S T)^{2} z\right) \leq \varphi_{2}\left(\varphi_{1}\left(\rho_{\alpha_{0}}(z, S T z)\right)\right) \leq \varphi_{1}\left(\rho_{\alpha_{0}}(z, S T z)\right)<\rho_{\alpha_{0}}(z, S T z) .
$$

On the other hand, by (3.13) and (3.14), we can prove that $\left\{\rho_{\alpha_{0}}\left(x_{n}, x_{n+1}\right)\right\}$ and $\left\{\tilde{\rho}_{\alpha_{0}}\left(y_{n}, y_{n+1}\right)\right\}$ are non-increasing. In fact, applying (3.14), we obtain

$$
\begin{aligned}
& G\left(\rho_{\alpha_{0}}\left(S y_{n}, S T x_{n}\right), \tilde{\rho}_{\alpha_{0}}\left(y_{n}, T x_{n}\right), \rho_{\alpha_{0}}\left(x_{n}, S y_{n}\right), \rho_{\alpha_{0}}\left(x_{n}, S T x_{n}\right)\right) \\
& \quad=G\left(\rho_{\alpha_{0}}\left(S\left(T x_{n-1}\right), S T x_{n}\right), \tilde{\rho}_{\alpha_{0}}\left(T x_{n-1}, T x_{n}\right), \rho_{\alpha_{0}}\left(x_{n}, x_{n}\right), \rho_{\alpha_{0}}\left(x_{n}, S T x_{n}\right)\right) \\
& \quad=G\left(\rho_{\alpha_{0}}\left(x_{n}, x_{n+1}\right), \widetilde{\rho}_{\alpha_{0}}\left(y_{n}, y_{n+1}\right), 0, \rho_{\alpha_{0}}\left(x_{n}, x_{n+1}\right)\right) \leq 0 .
\end{aligned}
$$


Note that $G \in \mathcal{A}_{\varphi_{2}}$, we have

$$
\rho_{\alpha_{0}}\left(x_{n}, x_{n+1}\right) \leq \varphi_{2}\left(\widetilde{\rho}_{\alpha_{0}}\left(y_{n}, y_{n+1}\right)\right) \leq \widetilde{\rho}_{\alpha_{0}}\left(y_{n}, y_{n+1}\right)
$$

Similarly, by (3.13) and $F \in \mathcal{A}_{\varphi_{1}}$, we can obtain that

$$
\tilde{\rho}_{\alpha_{0}}\left(y_{n}, y_{n+1}\right) \leq \varphi_{1}\left(\rho_{\alpha_{0}}\left(x_{n}, x_{n-1}\right)\right) \leq \rho_{\alpha_{0}}\left(x_{n}, x_{n-1}\right) .
$$

Then from (3.18) and (3.19), we have $\rho_{\alpha_{0}}\left(x_{n}, x_{n+1}\right) \leq \rho_{\alpha_{0}}\left(x_{n}, x_{n-1}\right)$ and $\widetilde{\rho}_{\alpha_{0}}\left(y_{n}, y_{n+1}\right) \leq$ $\widetilde{\rho}_{\alpha_{0}}\left(y_{n-1}, y_{n}\right)$, i.e., $\left\{\rho_{\alpha_{0}}\left(x_{n}, x_{n+1}\right)\right\}$ and $\left\{\widetilde{\rho}_{\alpha_{0}}\left(y_{n}, y_{n+1}\right)\right\}$ are two non-increasing sequences, and so there exist $\xi, \eta \geq 0$ such that $\lim _{n \rightarrow \infty} \rho_{\alpha_{0}}\left(x_{n}, x_{n+1}\right)=\xi$ and $\lim _{n \rightarrow \infty} \tilde{\rho}_{\alpha_{0}}\left(y_{n}, y_{n+1}\right)=\eta$. Since $T$ and $S$ are continuous, by Lemma 2.6, we can obtain that

$$
\rho_{\alpha_{0}}(z, S T z)=\lim _{i \rightarrow \infty} \rho_{\alpha_{0}}\left(x_{n_{i}}, S T x_{n_{i}}\right)=\lim _{i \rightarrow \infty} \rho_{\alpha_{0}}\left(x_{n_{i}}, x_{n_{i}+1}\right)=\xi
$$

and

$$
\rho_{\alpha_{0}}\left(S T z,(S T)^{2} z\right)=\lim _{i \rightarrow \infty} \rho_{\alpha_{0}}\left(S T x_{n_{i}},(S T)^{2} x_{n_{i}}\right)=\lim _{i \rightarrow \infty} \rho_{\alpha_{0}}\left(x_{n_{i}+1}, x_{n_{i}+2}\right)=\xi,
$$

which imply that $\rho_{\alpha_{0}}\left(S T z,(S T)^{2} z\right)=\rho_{\alpha_{0}}(z, S T z)$. This is a contradiction with (3.17). Hence, $y_{*}$ is a fixed point of TS.

Now we set $S y_{*}=x_{*}$, then $y_{*}=T S y_{*}=T x_{*} \Rightarrow S T x_{*}=S y_{*}=x_{*}$, i.e., $x_{*}$ is a fixed point of ST.

Finally, we show the uniqueness of a fixed point. Assume that $y^{*}$ is another fixed point of $T S$ with $y_{*} \neq y^{*}$, then $S y_{*} \neq S y^{*}$. By (3.13), we have for each $\alpha \in(0,1]$

$$
\begin{gathered}
F\left(\widetilde{\rho}_{\alpha}\left(T S y_{*}, T S y^{*}\right), \rho_{\alpha}\left(S y_{*}, S y^{*}\right), \tilde{\rho}_{\alpha}\left(y^{*}, T S y_{*}\right), \tilde{\rho}_{\alpha}\left(y^{*}, T S y^{*}\right)\right) \\
\quad=F\left(\widetilde{\rho}_{\alpha}\left(y_{*}, y^{*}\right), \rho_{\alpha}\left(S y_{*}, S y^{*}\right), \tilde{\rho}_{\alpha}\left(y^{*}, y_{*}\right), 0\right) \leq 0 .
\end{gathered}
$$

Note that $F \in \mathcal{A}_{\varphi_{1}}$, it is not difficult to obtain that $\tilde{\rho}_{\alpha}\left(y_{*}, y^{*}\right) \leq \varphi_{1}\left(\rho_{\alpha}\left(S y_{*}, S y^{*}\right)\right)$ for each $\alpha \in(0,1]$. Since $S y_{*} \neq S y^{*}$, there exists $\alpha_{0} \in(0,1]$ such that $\rho_{\alpha_{0}}\left(S y_{*}, S y^{*}\right)>0$. From $\varphi_{1} \in(\Phi)$, it follows that

$$
\tilde{\rho}_{\alpha_{0}}\left(y_{*}, y^{*}\right) \leq \varphi_{1}\left(\rho_{\alpha_{0}}\left(S y_{*}, S y^{*}\right)\right)<\rho_{\alpha_{0}}\left(S y_{*}, S y^{*}\right)
$$

On the other hand, by $y_{*} \neq T S y^{*}$, from (3.14) and $G \in \mathcal{A}_{\varphi_{2}}$, it is easy to obtain that $\rho_{\alpha_{0}}\left(S y_{*}, S y^{*}\right) \leq \varphi_{2}\left(\widetilde{\rho}_{\alpha_{0}}\left(y_{*}, y^{*}\right)\right) \leq \widetilde{\rho}_{\alpha_{0}}\left(y_{*}, y^{*}\right)$. This is a contradiction with (3.20). Hence, the uniqueness of a fixed point for TS is true.

Assume that $x^{*}$ is also a fixed point of $S T$. Let $\bar{y}=T x^{*}$, then $T S \bar{y}=T S T x^{*}=T x^{*}=\bar{y}$. By the uniqueness of a fixed point for $T S$, we know $\bar{y}=y_{*}$. This shows that $x_{*}=S y_{*}=S \bar{y}=$ $S T x^{*}=x^{*}$, i.e., the uniqueness of a fixed point for $S T$ holds. This completes the proof.

Let $(X, \rho)$ be a metric space and

$$
d(x, y)(t)=\left\{\begin{array}{ll}
1, & t=\rho(x, y), \\
0, & t \neq \rho(x, y),
\end{array} \quad \text { for all } x, y \in X \text { and } t \in \mathbb{R} .\right.
$$


Then $(X, d, \min , \max )$ is a FMS $(c f .[1,4])$. It is easy to see that $(X, \rho)$ and $(X, d, \min$, max $)$ are homeomorphic and $\rho(x, y)=\rho_{\alpha}(x, y)$ for all $\alpha \in(0,1]$.

Theorem 3.4 Let $(X, \rho)$ and $(Y, \widetilde{\rho})$ be two complete metric spaces. Let $T: X \rightarrow Y$ and $S: Y \rightarrow X$ be two non-self mappings, and $\varphi_{1}, \varphi_{2} \in\left(\Phi_{0}\right)$. If there exist $F \in \mathcal{A}_{\varphi_{1}}$ and $G \in \mathcal{A}_{\varphi_{2}}$ such that

$$
\begin{aligned}
& F(\widetilde{\rho}(T x, T S y), \rho(x, S y), \widetilde{\rho}(y, T x), \widetilde{\rho}(y, T S y)) \leq 0, \\
& G(\rho(S y, S T x), \widetilde{\rho}(y, T x), \rho(x, S y), \rho(x, S T x)) \leq 0
\end{aligned}
$$

for all $x \in X, y \in Y$, then $S T$ has a unique fixed point $x_{*} \in X$, and TS has a unique fixed point $y_{*} \in Y$ with $T x_{*}=y_{*}, S y_{*}=x_{*}$.

Proof Note that the topology and completeness of $(X, \rho)$ and $(Y, \widetilde{\rho})$, and the induced FMS $(X, d, \min , \max )$ and FMS $(Y, \tilde{d}, \min , \max )$ are coincident respectively, as well as $\rho\left(x_{1}, x_{2}\right)=$ $\rho_{\alpha}\left(x_{1}, x_{2}\right)$ for all $x_{1}, x_{2} \in X$ and $\alpha \in(0,1]$, and $\widetilde{\rho}\left(y_{1}, y_{2}\right)=\widetilde{\rho}_{\alpha}\left(y_{1}, y_{2}\right)$ for all $y_{1}, y_{2} \in Y$ and $\alpha \in(0,1]$. Then it is not difficult to see that inequality (3.1) holds as a result of (3.21), and inequality (3.2) holds as a result of (3.22). Moreover, the other conditions of Theorem 3.1 are satisfied, thus by Theorem 3.1, the theorem is proved.

Applying the same method, we can obtain the following theorem by virtue of Theorem 3.3.

Theorem 3.5 Let $(X, \rho)$ and $(Y, \widetilde{\rho})$ be two complete metric spaces. Let $\varphi_{1}, \varphi_{2} \in(\Phi)$. Suppose that $T: X \rightarrow Y$ and $S: Y \rightarrow X$ are two continuous non-self mappings satisfying the following conditions:

(1) There exists $F \in \mathcal{A}_{\varphi_{1}}$ such that

$$
F(\widetilde{\rho}(T x, T S y), \rho(x, S y), \widetilde{\rho}(y, T x), \widetilde{\rho}(y, T S y)) \leq 0
$$

for all $x \in X, y \in Y$ with $x \neq$ Sy;

(2) There exists $G \in \mathcal{A}_{\varphi_{2}}$ such that

$$
G(\rho(S y, S T x), \widetilde{\rho}(y, T x), \rho(x, S y), \rho(x, S T x)) \leq 0
$$

for all $x \in X, y \in Y$ with $y \neq T x$;

(3) There exists $x_{0} \in X$ such that $\left\{(S T)^{n} x_{0}\right\}$ has an accumulation point in $X$.

Then ST has a unique fixed point $x_{*}$ in $X$, and TS has a unique fixed point $y_{*}$ in $Y$ with $T x_{*}=y_{*}, S y_{*}=x_{*}$.

Remark 3.2 Taking $\varphi(t)=c t, c \in(0,1), t \geq 0$ and $F, G \in \mathcal{F}$ in Theorems 3.4 and 3.5, we can obtain Theorems 3 and 4 in [13], respectively. This shows that our results improve and generalize Theorems $3-4$ in [13] , and so the main results in $[11,12]$.

\section{Applications to Menger probabilistic metric spaces and example}

In this section, we first point out that our fixed point results for fuzzy metric spaces contain some corresponding results for Menger probabilistic metric spaces. After that, we give an example to discuss the validity of the hypotheses of Theorem 3.1. 
Definition 4.1 A function $F: R \rightarrow[0,1]$ is called a distribution function if it is nondecreasing and left-continuous with $\inf _{t \in \mathbb{R}} F(t)=0$ and $\sup _{t \in \mathbb{R}} F(t)=1$.

If $F$ is a distribution function which satisfies $F(0)=0$, then $F$ is called a nonnegative distribution function. Let $\mathcal{F}^{+}$be the set of all nonnegative distribution functions. A special element of $\mathcal{F}^{+}$is the Heaviside function $H$ defined by

$$
H(t)= \begin{cases}1, & t>0 \\ 0, & t \leq 0 .\end{cases}
$$

Definition 4.2 (Hadžić and Pap [19]) A function $\Delta:[0,1] \times[0,1] \rightarrow[0,1]$ is called a triangular norm (for short, a $t$-norm) if the following conditions are satisfied for any $a, b, c, d \in[0,1]$ :

$(\triangle-1) \quad \triangle(a, 1)=a$

$(\triangle-2) \quad \triangle(a, b)=\triangle(b, a)$

$(\triangle-3) \quad \triangle(a, b) \geq \triangle(c, d)$, for $a \geq c, b \geq d$;

$(\triangle-4) \triangle(\triangle(a, b), c)=\triangle(a, \triangle(b, c))$.

For each $a \in[0,1]$, the sequence $\left\{\Delta^{n}(a)\right\}_{n=1}^{\infty}$ is defined by $\Delta^{1}(a)=a$ and $\Delta^{n}(a)=$ $\triangle\left(\triangle^{n-1}(a), a\right)$. A $t$-norm $\triangle$ is said to be of $H$-type if the sequence of functions $\left\{\Delta^{n}(a)\right\}_{n=1}^{\infty}$ is equicontinuous at $a=1$.

Lemma 4.1 (Xiao et al. [10]) Let $\triangle$ be a t-norm for each $a, b \in[0,1], R$ be defined by $R(a, b)=1-\triangle(1-a, 1-b)$, then

(1) $R$ is a symmetric and nondecreasing function such that $R(1,1)=1$;

(2) If $\triangle$ is of H-type, then $R$ satisfies (R-2).

Remark 4.1 (Xiao etal. [10]) Let $R:[0,1] \times[0,1] \rightarrow[0,1]$ be a symmetric and nondecreasing function such that $R(1,1)=1$ and $\triangle_{0}(a, b)=1-R(1-a, 1-b)$ for all $a, b \in[0,1]$. Then $\triangle_{0}$ satisfies $(\triangle-2)$ and $(\triangle-3)$. But $\triangle_{0}$ does not necessarily satisfy $(\triangle-1)$ and $(\triangle-4)$. Hence $\triangle_{0}$ is not necessarily a $t$-norm. From Remark 2.1 we see that $R(s, t)=[\min \{s, t\}]^{1 / 2}$ satisfies (R-2); but $\triangle_{0}(s, t)=1-R(1-s, 1-t)=1-[1-\max \{s, t\}]^{1 / 2}$ is not a $t$-norm of $H$-type.

Definition 4.3 (Hadžić and Pap [19]) A triplet $(X, F, \triangle)$ is called a Menger probabilistic metric space if $X$ is a non-empty set, $\triangle$ is a $t$-norm and $F$ is a mapping from $X \times X$ into $\mathcal{F}^{+}$satisfying the following conditions $\left(F(x, y)\right.$ for $x, y \in X$ is denoted by $\left.F_{x, y}\right)$ :

(M-1) $F_{x, y}(t)=H(t)$ for all $t \in \mathbb{R}$ if and only if $x=y$;

(M-2) $F_{x, y}(t)=F_{y, x}(t)$ for all $x, y \in X$ and $t \in \mathbb{R}$;

(M-3) $F_{x, y}(t+s) \geq \triangle\left(F_{x, z}(t), F_{z, y}(s)\right)$ for all $x, y, z \in X$ and $t, s \in \mathbb{R}^{+}$.

Lemma 4.2 (Kaleva and Seikkala [1]) Let $(X, F, \triangle)$ be a Menger probabilistic metric space, $x, y \in X$ and $\omega_{x y}=\sup \left\{t: F_{x, y}(t)=0\right\}$. Let $d: X \times X \rightarrow G^{+}$be a mapping defined by

$$
d(x, y)(t)= \begin{cases}0, & t<\omega_{x, y} \\ 1-F_{x, y}(t), & t \geq \omega_{x, y} .\end{cases}
$$

Then $\omega_{x, y} \geq 0$ and $d(x, y) \in G^{+}$. Let $L, R:[0,1] \times[0,1] \rightarrow[0,1]$ be defined by $L \equiv 0$ and $R(a, b)=1-\triangle(1-a, 1-b)$. Then $(X, d, L, R)$ is $a$ FMS. 
From Lemma 4.2 we see that each Menger probabilistic metric space can be considered as a special Kaleva-Seikkala's type fuzzy metric space. But Remark 4.1 shows that in general a Kaleva-Seikkala's type fuzzy metric space cannot be considered as a Menger probabilistic metric space. Hence, as direct consequences of our results, we can obtain the corresponding fixed point theorems in Menger probabilistic metric spaces. For example, from Theorem 3.1 and Lemmas 4.1-4.2 we can obtain the following consequence.

Theorem 4.1 Let $(X, F, \triangle)$ and $(Y, \widetilde{F}, \widetilde{\triangle})$ be two complete Menger probabilistic metric spaces such that $\triangle$ and $\widetilde{\triangle}$ are two t-norms of H-type. Let $T: X \rightarrow Y$ and $S: Y \rightarrow X$ be two non-self mappings, and $\varphi_{1}, \varphi_{2} \in\left(\Phi_{0}\right)$. If there exist $f \in \mathcal{A}_{\varphi_{1}}$ and $g \in \mathcal{A}_{\varphi_{2}}$ such that

$$
\begin{aligned}
& \widetilde{F}_{T x, T S y}(u-f(u, v, w, s)) \geq \min \left\{\widetilde{F}_{T x, T S y}(u), F_{x, S y}(v), \widetilde{F}_{y, T x}(w), \widetilde{F}_{y, T S y}(s)\right\}, \\
& F_{S y, S T x}(u-g(u, v, w, s)) \geq \min \left\{F_{S y, S T x}(u), \widetilde{F}_{y, T x}(v), F_{x, S y}(w), F_{x, S T x}(s)\right\}
\end{aligned}
$$

for all $x \in X, y \in Y$ and $u, v, w, s \geq 0$, then ST has a unique fixed point $x_{*} \in X$, and TS has a unique fixed point $y_{*} \in Y$ with $T x_{*}=y_{*}, S y_{*}=x_{*}$.

Proof Let $(X, d, L, R)$ and $(Y, \widetilde{d}, \widetilde{L}, \widetilde{R})$ be defined as in Lemma 4.2, respectively. Then $(X, d, L, R)$ and $(Y, \widetilde{d}, \widetilde{L}, \widetilde{R})$ are two FMSs. Since $\Delta$ and $\widetilde{\Delta}$ are of $H$-type, by Lemma 4.1, $R$ and $\widetilde{R}$ satisfy (R-2). Now we check that (3.1) holds.

From (4.1) we see that

$$
\begin{aligned}
& F_{x, y}(t)>1-\alpha \Leftrightarrow d(x, y)(t)<\alpha \quad \Leftrightarrow \quad \rho_{\alpha}(x, y)<t, \\
& \quad \text { for all } x, y \in X \text { and } \alpha \in(0,1] .
\end{aligned}
$$

For each $x \in X, y \in Y$ and $\alpha \in(0,1]$, if we set $\tilde{\rho}_{\alpha}(T x, T s y)=u, \rho_{\alpha}(x, S y)=v, \tilde{\rho}_{\alpha}(y, T x)=w$, $\tilde{\rho}_{\alpha}(y, T S y)=s$, then for any $\varepsilon>0$, it is obvious that $\tilde{\rho}_{\alpha}(T x, T s y)<u+\varepsilon, \rho_{\alpha}(x, S y)<v+\varepsilon$, $\tilde{\rho}_{\alpha}(y, T x)<w+\varepsilon, \widetilde{\rho}_{\alpha}(y, T S y)<s+\varepsilon$. By $(4.4)$, we have $\widetilde{F}_{T x, T S y}(u+\varepsilon)>1-\alpha, F_{x, S y}(v+\varepsilon)>1-\alpha$, $\widetilde{F}_{y, T x}(w+\varepsilon)>1-\alpha, \widetilde{F}_{y, T S y}(s+\varepsilon)>1-\alpha$. Note (4.2), we can obtain that

$$
\widetilde{F}_{T x, T S y}(u+\varepsilon-f(u+\varepsilon, v+\varepsilon, w+\varepsilon, s+\varepsilon))>1-\alpha,
$$

which implies that

$$
\tilde{\rho}_{\alpha}(T x, T S y)=u<u+\varepsilon-f(u+\varepsilon, v+\varepsilon, w+\varepsilon, s+\varepsilon),
$$

i.e.,

$$
f\left(\widetilde{\rho}_{\alpha}(T x, T S y)+\varepsilon, \rho_{\alpha}(x, S y)+\varepsilon, \tilde{\rho}_{\alpha}(y, T x)+\varepsilon, \tilde{\rho}_{\alpha}(y, T S y)+\varepsilon\right)<\varepsilon .
$$

Then by the arbitrariness of $\varepsilon$ and the right-lower semi-continuity of $f$, we have

$$
\begin{aligned}
& f\left(\widetilde{\rho}_{\alpha}(T x, T S y), \rho_{\alpha}(x, S y), \widetilde{\rho}_{\alpha}(y, T x), \tilde{\rho}_{\alpha}(y, T S y)\right) \\
& \quad \leq \liminf _{\varepsilon \rightarrow 0^{+}} f\left(\widetilde{\rho}_{\alpha}(T x, T S y)+\varepsilon, \rho_{\alpha}(x, S y)+\varepsilon, \widetilde{\rho}_{\alpha}(y, T x)+\varepsilon, \widetilde{\rho}_{\alpha}(y, T S y)+\varepsilon\right) \\
& \quad \leq 0
\end{aligned}
$$

for each $x \in X, y \in Y$ and $\alpha \in(0,1]$, i.e., (3.1) holds for all $x, y \in X$ and $\alpha \in(0,1]$. 
Similarly, by (4.3), we can prove that (3.2) also holds. Moreover, the other conditions in Theorem 3.1 are satisfied, thus by Theorem 3.1, the theorem is proved.

In Theorem 4.1, taking $(X, F, \triangle)=(Y, \widetilde{F}, \widetilde{\triangle}), S=T$, we obtain the following corollary.

Corollary 4.1 Let $(X, F, \triangle)$ be a complete Menger probabilistic metric space such that $\triangle$ is a $t$-norm of H-type. Let $T: X \rightarrow X$ be a self-mapping, and $\varphi \in\left(\Phi_{0}\right)$. If there exists $f \in \mathcal{A}_{\varphi}$ such that

$$
F_{T x, T^{2} y}(u-f(u, v, w, s)) \geq \min \left\{F_{T x, T^{2} y}(u), F_{x, T y}(v), F_{y, T x}(w), F_{y, T^{2} y}(s)\right\}
$$

for all $x, y \in X$ and $u, v, w, s \geq 0$, then $T$ has a unique fixed point in $X$.

Corollary 4.2 Let $(X, F, \triangle)$ be a complete Menger probabilistic metric space such that $\triangle$ is a $t$-norm of H-type. Let $T: X \rightarrow X$ be a self-mapping. If there exists $\varphi \in\left(\Phi_{0}\right)$ such that

$$
F_{T x, T y}(\varphi(v)) \geq F_{x, y}(v)
$$

for all $x \in X, y \in T(X)$ and $v \geq 0$, then $T$ has a unique fixed point in $X$.

Proof Taking $f\left(t_{1}, t_{2}, t_{3}, t_{4}\right)=t_{1}-\varphi\left(\lambda_{1} t_{2}+\lambda_{2} t_{3}+\lambda_{3} t_{4}\right), \lambda_{1}=1, \lambda_{2}=\lambda_{3}=0$, from Example 2, we obtain $f=F_{3} \in \mathcal{A}_{\varphi}$. Furthermore, for all $x, y \in X$ and $u, v, w, s \geq 0$, by $T y \in T(X)$ and (4.6), we have

$$
\begin{aligned}
F_{T x, T^{2} y}(\varphi(v)) & =F_{T x, T^{2} y}(u-(u-\varphi(v))) \geq F_{x, T y}(v) \\
& \geq \min \left\{F_{T x, T^{2} y}(u), F_{x, T y}(v), F_{y, T x}(w), F_{y, T^{2} y}(s)\right\},
\end{aligned}
$$

which implies that (4.5) holds. Therefore, the conclusion follows from Corollary 4.1 immediately.

Remark 4.2 Recently, in [20] Jachymski obtained the following result.

Theorem J Let $(X, F, \triangle)$ be a complete Menger probabilistic metric space such that $\triangle$ is a continuous $t$-norm of $H$-type. Let a function $\varphi: \mathbb{R}^{+} \rightarrow \mathbb{R}^{+}$be such that, for any $r>0$,

$$
0<\varphi(r)<r \quad \text { and } \quad \lim _{n \rightarrow \infty} \varphi^{n}(r)=0
$$

Let $T: X \rightarrow X$ be a mapping such that

$$
F_{T x, T y}(\varphi(t)) \geq F_{x, y}(t) \quad \text { for all } t>0 \text { and } \quad x, y \in X
$$

Then there exists a unique $x_{*} \in X$ such that $T x_{*}=x_{*}$.

Remark 4.3 Comparing Corollary 4.2 with Theorem J, it is not difficult to see the differences between them. Although Corollary 4.2 demands the function $\varphi \in\left(\Phi_{0}\right)$ to imply that (4.7) holds by Definition 2.4(2), it does not require the $t$-norm of $H$-type to be continuous for all $y \in X$ such that (4.8) holds. 
Finally, we give an example to support the main results presented herein.

Example 5 Suppose that $X=[-1,1] \subset \mathbb{R}$. Define $d: X \times X \rightarrow G^{+}$by

$$
d(x, y)(t)= \begin{cases}0, & \text { if } t<0, \\ 1, & \text { if } t=0, \quad \text { for all } x, y \in X . \\ 1-e^{-\frac{|x-y|}{t},}, & \text { if } t>0,\end{cases}
$$

Let $L, R:[0,1] \times[0,1] \rightarrow[0,1]$ be defined by $L \equiv 0$ and $R(a, b)=\max \{a, b\}$. Then $(X, d, L, R)$ is a complete FMS.

In fact, (FM-1), (FM-2) and (FM-3L) are easy to check. We only see (FM-3R). Since $d(x, y)(0)=1$ for all $x, y \in X$, and so $\lambda_{1}(x, y)=0$ for all $x, y \in X$. To prove (FM-3R), we assume that $s, t>0, x, y, z \in X$ and

$$
R(d(x, z)(t), d(z, y)(s))=\max \left\{1-e^{-\frac{|x-z|}{t}}, 1-e^{-\frac{|z-y|}{s}}\right\}=1-e^{-\frac{|x-z|}{t}} .
$$

Then we have $s|x-z| \geq t|z-y|$, and so $\frac{t+s}{t}|x-z|=|x-z|+\frac{s}{t}|x-z| \geq|x-z|+|z-y| \geq|x-y|$. It follows that

$$
d(x, y)(t+s)=1-e^{-\frac{|x-y|}{t+s}} \leq 1-e^{-\frac{|x-z|}{t}}=R(d(x, z)(t), d(z, y)(s)) .
$$

Hence (FM-3R) holds. It is clear that $(X, d, L, R)$ is complete.

In the same manner, if we take $Y=[1,3] \subset \mathbb{R}$ and $\tilde{d}: Y \times Y \rightarrow G^{+}$given by (4.9), then $(Y, \tilde{d}, L, R)$ is a complete FMS.

Define $T: X \rightarrow Y$ and $S: Y \rightarrow X$ by

$$
T x=\left\{\begin{array}{ll}
\frac{9}{8}, & x \in[-1,0), \\
\frac{11}{8}, & x \in[0,1],
\end{array} \text { and } \quad S y= \begin{cases}\frac{5}{8}, & y \in\left[1, \frac{17}{16}\right) \\
\frac{7}{8}, & y \in\left[\frac{17}{16}, 3\right] .\end{cases}\right.
$$

Obviously, $S T x=\frac{7}{8}$ for each $x \in[-1,1], T S y=\frac{11}{8}$ for each $y \in[1,3]$, and $S T\left(\frac{7}{8}\right)=\frac{7}{8}$, $T S\left(\frac{11}{8}\right)=\frac{11}{8}, T\left(\frac{7}{8}\right)=\frac{11}{8}, S\left(\frac{11}{8}\right)=\frac{7}{8}$.

Next we check that $T$ and $S$ satisfy the conditions in Theorem 3.1. Obviously, we have

$$
\rho_{\alpha}(x, y)=\left\{\begin{array}{ll}
0, & \text { if } \alpha=1, \\
-\frac{|x-y|}{\ln (1-\alpha)}, & \text { if } \alpha \in(0,1),
\end{array} \quad \text { for all } x, y \in X ;\right.
$$

and

$$
\tilde{\rho}_{\alpha}(x, y)=\left\{\begin{array}{ll}
0, & \text { if } \alpha=1, \\
-\frac{|x-y|}{\ln (1-\alpha)}, & \text { if } \alpha \in(0,1),
\end{array} \quad \text { for all } x, y \in Y .\right.
$$

We set $F(u, v, w, s)=u-\varphi_{1}(\max \{v, w, s\}), G(u, v, w, s)=u-\varphi_{2}(\max \{v, w, s\})$, where $\varphi_{1}(t)=\frac{1}{2} t$, $\varphi_{2}(t)=\frac{4}{5} t(\forall t \in \mathbb{R})$. It is easily seen that $\varphi_{1}, \varphi_{2} \in\left(\Phi_{0}\right), F \in \mathcal{A}_{\varphi_{1}}$ and $G \in \mathcal{A}_{\varphi_{2}}$. If $\alpha=1$, then (3.1) and (3.2) are easy to check. We only see $\alpha \in(0,1)$. In the next step, we consider the following four cases. 
Case 1. If $x \in[-1,0)$ and $y \in\left[1, \frac{17}{16}\right)$, then for each $\alpha \in(0,1)$ we have

$$
\begin{aligned}
& \tilde{\rho}_{\alpha}(T x, T S y)=\widetilde{\rho}_{\alpha}\left(\frac{9}{8}, \frac{11}{8}\right)=-\frac{1}{4 \ln (1-\alpha)}, \quad \rho_{\alpha}(x, S y)=\rho_{\alpha}\left(x, \frac{5}{8}\right)=-\frac{\left|x-\frac{5}{8}\right|}{\ln (1-\alpha)}, \\
& \tilde{\rho}_{\alpha}(y, T x)=\tilde{\rho}_{\alpha}\left(y, \frac{9}{8}\right)=-\frac{\left|y-\frac{9}{8}\right|}{\ln (1-\alpha)}, \quad \tilde{\rho}_{\alpha}(y, T S y)=\tilde{\rho}_{\alpha}\left(y, \frac{11}{8}\right)=-\frac{\left|y-\frac{11}{8}\right|}{\ln (1-\alpha)} .
\end{aligned}
$$

Since $\inf _{x \in[-1,0), y \in\left[1, \frac{17}{16}\right)}\left\{\max \left\{\left|x-\frac{5}{8}\right|,\left|y-\frac{9}{8}\right|,\left|y-\frac{11}{8}\right|\right\}\right\}=\frac{5}{8}$, i.e., $\frac{1}{4}-\frac{1}{2} \max \left\{\left|x-\frac{5}{8}\right|,\left|y-\frac{9}{8}\right|, \mid y-\right.$ $\left.\frac{11}{8} \mid\right\} \leq 0$ for all $x \in[-1,0)$ and $y \in\left[1, \frac{17}{16}\right)$, it follows that for each $\alpha \in(0,1)$

$$
-\frac{1}{4 \ln (1-\alpha)}-\frac{1}{2} \max \left\{-\frac{\left|x-\frac{5}{8}\right|}{\ln (1-\alpha)},-\frac{\left|y-\frac{9}{8}\right|}{\ln (1-\alpha)},-\frac{\left|y-\frac{11}{8}\right|}{\ln (1-\alpha)}\right\} \leq 0,
$$

which implies that (3.1) holds. Similarly, we have

$$
\begin{aligned}
& \rho_{\alpha}(S y, S T x)=\rho_{\alpha}\left(\frac{5}{8}, \frac{7}{8}\right)=-\frac{1}{4 \ln (1-\alpha)}, \quad \tilde{\rho}_{\alpha}(y, T x)=\tilde{\rho}_{\alpha}\left(y, \frac{9}{8}\right)=-\frac{\left|y-\frac{9}{8}\right|}{\ln (1-\alpha)}, \\
& \rho_{\alpha}(x, S y)=\rho_{\alpha}\left(x, \frac{5}{8}\right)=-\frac{\left|x-\frac{5}{8}\right|}{\ln (1-\alpha)}, \quad \rho_{\alpha}(x, S T x)=\rho_{\alpha}\left(x, \frac{7}{8}\right)=-\frac{\left|x-\frac{7}{8}\right|}{\ln (1-\alpha)} .
\end{aligned}
$$

Since $\inf _{x \in[-1,0), y \in\left[1, \frac{17}{16}\right)}\left\{\max \left\{\left|y-\frac{9}{8}\right|,\left|x-\frac{5}{8}\right|,\left|x-\frac{7}{8}\right|\right\}\right\}=\frac{7}{8}$, i.e., $\frac{1}{4}-\frac{4}{5} \max \left\{\left|y-\frac{9}{8}\right|,\left|x-\frac{5}{8}\right|, \mid x-\right.$ $\left.\frac{7}{8} \mid\right\} \leq 0$ for all $x \in[-1,0)$ and $y \in\left[1, \frac{17}{16}\right)$, it follows that for each $\alpha \in(0,1)$

$$
-\frac{1}{4 \ln (1-\alpha)}-\frac{4}{5} \max \left\{-\frac{\left|y-\frac{9}{8}\right|}{\ln (1-\alpha)},-\frac{\left|x-\frac{5}{8}\right|}{\ln (1-\alpha)},-\frac{\left|x-\frac{7}{8}\right|}{\ln (1-\alpha)}\right\} \leq 0,
$$

which implies that (3.2) also holds.

Case 2. If $x \in[-1,0)$ and $y \in\left[\frac{17}{16}, 3\right]$, then for each $\alpha \in(0,1)$ we have

$$
\begin{aligned}
& \tilde{\rho}_{\alpha}(T x, T S y)=\tilde{\rho}_{\alpha}\left(\frac{9}{8}, \frac{11}{8}\right)=-\frac{1}{4 \ln (1-\alpha)}, \quad \rho_{\alpha}(x, S y)=\rho_{\alpha}\left(x, \frac{7}{8}\right)=-\frac{\left|x-\frac{7}{8}\right|}{\ln (1-\alpha)}, \\
& \tilde{\rho}_{\alpha}(y, T x)=\tilde{\rho}_{\alpha}\left(y, \frac{9}{8}\right)=-\frac{\left|y-\frac{9}{8}\right|}{\ln (1-\alpha)}, \quad \tilde{\rho}_{\alpha}(y, T S y)=\tilde{\rho}_{\alpha}\left(y, \frac{11}{8}\right)=-\frac{\left|y-\frac{11}{8}\right|}{\ln (1-\alpha)} .
\end{aligned}
$$

Since $\inf _{x \in[-1,0), y \in\left[\frac{17}{16}, 3\right]}\left\{\max \left\{\left|x-\frac{7}{8}\right|,\left|y-\frac{9}{8}\right|,\left|y-\frac{11}{8}\right|\right\}\right\}=\frac{7}{8}$, i.e., $\frac{1}{4}-\frac{1}{2} \max \left\{\left|x-\frac{7}{8}\right|,\left|y-\frac{9}{8}\right|, \mid y-\right.$ $\left.\frac{11}{8} \mid\right\} \leq 0$ for all $x \in[-1,0)$ and $y \in\left[\frac{17}{16}, 3\right]$, it follows that for each $\alpha \in(0,1)$

$$
-\frac{1}{4 \ln (1-\alpha)}-\frac{1}{2} \max \left\{-\frac{\left|x-\frac{7}{8}\right|}{\ln (1-\alpha)},-\frac{\left|y-\frac{9}{8}\right|}{\ln (1-\alpha)},-\frac{\left|y-\frac{11}{8}\right|}{\ln (1-\alpha)}\right\} \leq 0,
$$

which implies that (3.1) holds. Similarly, we have $\rho_{\alpha}(S y, S T x)=\rho_{\alpha}\left(\frac{7}{8}, \frac{7}{8}\right)=0$, which shows that (3.2) also holds. 
Case 3. If $x \in[0,1]$ and $y \in\left[1, \frac{17}{16}\right)$, then for each $\alpha \in(0,1)$ we have $\widetilde{\rho}_{\alpha}(T x, T S y)=$ $\tilde{\rho}_{\alpha}\left(\frac{11}{8}, \frac{11}{8}\right)=0$, which shows that $(3.1)$ holds. In addition, for each $\alpha \in(0,1)$ we have

$$
\begin{aligned}
& \rho_{\alpha}(S y, S T x)=\rho_{\alpha}\left(\frac{5}{8}, \frac{7}{8}\right)=-\frac{1}{4 \ln (1-\alpha)}, \quad \tilde{\rho}_{\alpha}(y, T x)=\rho_{\alpha}\left(y, \frac{11}{8}\right)=-\frac{\left|y-\frac{11}{8}\right|}{\ln (1-\alpha)}, \\
& \rho_{\alpha}(x, S y)=\rho_{\alpha}\left(x, \frac{5}{8}\right)=-\frac{\left|x-\frac{5}{8}\right|}{\ln (1-\alpha)}, \quad \rho_{\alpha}(x, S T x)=\rho_{\alpha}\left(x, \frac{7}{8}\right)=-\frac{\left|x-\frac{7}{8}\right|}{\ln (1-\alpha)} .
\end{aligned}
$$

Since $\inf _{x \in[0,1], y \in\left[1, \frac{17}{16}\right)}\left\{\max \left\{\left|y-\frac{11}{8}\right|,\left|x-\frac{5}{8}\right|,\left|x-\frac{7}{8}\right|\right\}\right\}=\frac{5}{16}$, i.e., $\frac{1}{4}-\frac{4}{5} \max \left\{\left|y-\frac{11}{8}\right|,\left|x-\frac{5}{8}\right|, \mid x-\right.$ $\left.\frac{7}{8} \mid\right\} \leq 0$ for all $x \in[0,1]$ and $y \in\left[1, \frac{17}{16}\right)$, then we can obtain that for each $\alpha \in(0,1)$

$$
-\frac{1}{4 \ln (1-\alpha)}-\frac{4}{5} \max \left\{-\frac{\left|y-\frac{11}{8}\right|}{\ln (1-\alpha)},-\frac{\left|x-\frac{5}{8}\right|}{\ln (1-\alpha)},-\frac{\left|x-\frac{7}{8}\right|}{\ln (1-\alpha)}\right\} \leq 0,
$$

which implies that (3.2) also holds.

Case 4. If $x \in[0,1]$ and $y \in\left[\frac{17}{16}, 3\right]$, then for each $\alpha \in(0,1)$ we have $\widetilde{\rho}_{\alpha}(T x, T S y)=$ $\tilde{\rho}_{\alpha}\left(\frac{11}{8}, \frac{11}{8}\right)=0, \rho_{\alpha}(S y, S T x)=\rho_{\alpha}\left(\frac{7}{8}, \frac{7}{8}\right)=0$, which imply that (3.1) and (3.2) hold.

Thus, all the conditions of Theorem 3.1 are satisfied. This shows the validity of the hypotheses of our main results.

\section{Competing interests}

The authors declare that they have no competing interests.

\section{Authors' contributions}

All authors read and approved the final manuscript.

\section{Acknowledgements}

This work was supported by the Natural Science Foundation of the Jiangsu Higher Education Institutions (Grant no. 13KJB1 10004) and Qing Lan Project of Jiangsu Province of China.

Received: 7 June 2013 Accepted: 28 August 2013 Published: 07 Nov 2013

\section{References}

1. Kaleva, O, Seikkala, S: On fuzzy metric spaces. Fuzzy Sets Syst. 12, 215-229 (1984)

2. Hadžić, O: Fixed point theorems for multi-valued mappings in some classes of fuzzy metric spaces. Fuzzy Sets Syst. 29, 115-125 (1989)

3. Hadžić, O, Pap, E: A fixed point theorem for multivalued mappings in probabilistic metric spaces and an application in fuzzy metric spaces. Fuzzy Sets Syst. 127, 333-344 (2002)

4. Fang, J-X: A note on fixed point theorems of Hadžić. Fuzzy Sets Syst. 48, 391-395 (1992)

5. Jung, JS, Cho, YJ, Kang, SM, Chang, SS: Coincidence theorems for set-valued mappings and Ekeland's variational principle in fuzzy metric spaces. Fuzzy Sets Syst. 79, 239-250 (1996)

6. Zhu, J, Zhong, CK, Wang, GP: An extension of Ekeland's variational principle in fuzzy metric space and its applications. Fuzzy Sets Syst. 108, 353-363 (1999)

7. Zhu, J, Zhong, CK, Wang, GP: Vector-valued variational principle in fuzzy metric space and it's applications. Fuzzy Sets Syst. 119, 343-354 (2001)

8. Grabiec, M: Fixed points in fuzzy metric spaces. Fuzzy Sets Syst. 27, 385-389 (1988)

9. Huang, H, Wu, C-X: On the completion of fuzzy metric spaces. Fuzzy Sets Syst. 159, 2596-2605 (2008)

10. Xiao, J-Z, Zhu, X-H, Jin, X: Fixed point theorems for nonlinear contractions in Kaleva-Seikkala's type fuzzy metric spaces. Fuzzy Sets Syst. 200, 65-83 (2012)

11. Fisher, B: Fixed point on two metric spaces. Glas. Mat. 16(36), 333-337 (1981)

12. Telci, M: Fixed points on two complete and compact metric spaces. Appl. Math. Mech. 22(5), 564-568 (2001)

13. Aliouche, A, Fisher, B: Fixed point theorems for mappings satisfying an implicit relation on two complete and compact metric spaces. Appl. Math. Mech. 27(9), 1065-1070 (2006)

14. Dubois, D, Prade, H: Operations on fuzzy numbers. Int. J. Syst. Sci. 9(6), 613-626 (1978)

15. Bag, T, Samanta, SK: Fuzzy bounded linear operators in Felbin's type fuzzy normed. Fuzzy Sets Syst. 159, 685-707 (2008)

16. Xiao, J-Z, Zhu, X-H: On linearly topological structure and property of fuzzy normed linear space. Fuzzy Sets Syst. 125 , 153-161 (2002)

17. Xiao, J-Z, Zhu, X-H: Topological degree theory and fixed point theorems in fuzzy normed linear spaces. Fuzzy Sets Syst. $147,437-452(2004)$ 
18. Boyd, DW, Wong, JS: On nonlinear contractions. Proc. Am. Math. Soc. 20, 458-464 (1969)

19. Hadžić, O, Pap, E: Fixed Point Theory in Probabilistic Metric Spaces. Mathematics and Its Applications, vol. 536. Kluwer Academic, Dordrecht (2001)

20. Jachymski, J: On probabilistic $\varphi$-contractions on Menger spaces. Nonlinear Anal. TMA 73, 2199-2203 (2010)

10.1186/1687-1812-2013-254

Cite this article as: Song and Wang: Fixed point theorems for complex non-self mappings satisfying an implicit

relation in Kaleva-Seikkala's type fuzzy metric spaces. Fixed Point Theory and Applications 2013, 2013:254

Submit your manuscript to a SpringerOpen ${ }^{\odot}$ journal and benefit from:

- Convenient online submission

Rigorous peer review

Immediate publication on acceptance

- Open access: articles freely available online

- High visibility within the field

- Retaining the copyright to your article

Submit your next manuscript at $>$ springeropen.com 